\title{
The Impact of Selecting Different Airport Sites on the Urban Heat Island Effect: a Case Study of Dalian, China
}

\author{
Tiaolan $\mathrm{Yu}^{1, \text { a }}$, Tianlan Zhou ${ }^{1, \mathrm{~b}}$, Nuo Wang ${ }^{2, \mathrm{c}}$ \\ ${ }^{1}$ College of Transport \& Communications, Shanghai Maritime University, Shanghai 201306, China \\ ${ }^{2}$ Department of Transportation Management, Dalian Maritime University, Dalian 116026, China
}

\begin{abstract}
Keywords: Heat Island Effect; Land Surface Temperature; Remote Sensing; Airport Site Selection Abstract. To study the influence of selecting different airport sites on the urban heat island effect, we retrieved the land surface temperature (LST) of Dalian's main urban area using the thermal infrared band from the Landsat- 8 data and graded thermal field. The results show that the Dalian Zhou Shuizi International Airport in Dalian's main urban area belongs to the high temperature zone, which indicates that the airport is an important contributor to the urban heat island effect. Similarly, we studied the urban area of Osaka, Japan, and found that the average land surface temperature of Kansai International Airport, $5 \mathrm{~km}$ from the coastline, is far lower than the average temperature of Osaka Airport in Osaka's urban area. This result shows that an offshore artificial island airport can effectively reduce the urban heat island effect compared to a terrestrial airport, which is more favorable for the ecological environment of the city.
\end{abstract}

\section{Introduction}

In recent decades, with increasing concern over urban ecological environment change the urban heat island (UHI) effect has drawn widespread attention. The UHI phenomenon is defined as surface and atmospheric modifications due to urbanisation that generally lead to a modified thermal climate that is warmer than the surrounding non-urbanised areas, particularly at night (Voogt et al., 2005 Pandey et al., 2012). Due to the UHI, the urban/rural temperature difference creates a convective circulation (Velazquez-Lozada et al., 2006), which can increase the air pollutant concentration. When the UHI effect is strong and the landscaping ratio is low, the ecological corridor function will be reduced such that fresh air cannot be transported to the urban region, which seriously affects the city's environmental quality (Velazquez-Lozada et al., 2006). Therefore, reducing the UHI effect is important to the urban environment.

Factors that contribute to the formation of the UHI include anthropogenic heat sources, aerosols from pollutants, and rapid water canalisation due to the presence of buildings and streets Nevertheless, the thermal inertia of concrete and the absence of vegetation in the urban areas are considered the most significant causes of the UHI (Velazquez-Lozada et al., 2006). Within a city, many artificial structures such as shop floors and building walls have changed the pad surface thermal properties. Artificial structures quickly absorb heat and have a small heat capacity; therefore, in the same solar radiation conditions, the natural underlying surfaces (e.g., green space, water) warm faster, and the natural surface temperatures in an urban environment will be significantly higher than in the rural environment. Yin et al. (2009) used Landsat ETM+ daytime remote sensing data of Shanghai to qualitatively and quantitatively analyse the relationships between the daytime ground brightness temperature (GBT) distribution and land use types of the city. The results showed that the anthropogenic establishment sections increased the GBT both in the summer and winter. Xiao et al. (2007) examined the effect of impervious surface (IS) spatial patterns on the LST in Beijing, China. The results of correlation analysis further showed that IS tended to be positively correlated with LST. Jusuf et al. (2007) investigated and identified land use types that have the greatest influence on the increase in ambient temperature in Singapore with remote sensing data and a geographical information system (GIS). The results show that land usage will influence the urban temperature, and the airport belongs to a high temperature zone. With the Normalized Difference Vegetation Index (NDVI), Normalized Difference Water Index (NDWI), Normalized Difference Bareness Index 
(NDBaI) and Normalized Difference Build-up Index (NDBI), Chen et al. (2006) quantitatively analysed the relationship between the UHI and land-cover changes in Shenzhen, China.

Site observation (Giannaros et al., 2012) and remote sensing are two main ways to investigate the urban heat effect. However, the site observation method is time-consuming and cannot obtain the land surface temperature (LST) fully and simultaneously. Many researchers have focused on using the thermal infrared band. Price et al. $(1979,1984)$ first described the relations between vegetation index, soil moisture and radiation temperature with a mathematical model. Carlson et al. $(1990,2002)$ studied the impact of urbanisation on tropical rainforests with remote sensing data in Costa Rica and San Jose. Streuker $(2002,2003)$ studied the UHI distribution and the relation between the UHI and suburban temperatures in Houston, USA. Gallo et al. (1993) first estimated the UHI and its impact on temperature variations between urban and suburban areas by using the vegetation index and found that the temperature has a significant linear relation with the vegetation index.

A civil aviation airport is a typical large-scale artificial structure that plays an important role in the urban heat island effect. From the perspective of improving the urban environment, the site selection of a civil aviation airport has to carefully consider the influence of the urban heat island effect. This paper used Landsat 8 remote sensing data to analyse the degree that a civil aviation airport contributes to the urban heat island effect by using a single-window surface temperature retrieved algorithm. Using the Dalian civil aviation airport expansion and site selection as an example, we analysed the influence of different airport site selections on the urban heat island effect, which can provide a scientific basis for weighing the relation between the urban environment and airport establishment.

\section{Study area and satellite data}

\section{Study area}

Dalian is a coastal city at the converging point of the Bohai Sea and Yellow Sea located on the southernmost tip of northeast China. Dalian Zhoushuizi International Airport (DIA), approximately $9.5 \mathrm{~km}$ from the city centre, covers an area of $2.84 \mathrm{~km}^{2}$ and has $0.135 \mathrm{~km}^{2}$ of airport terminal. DIA has a single 3,300 m runway for landing/take-off and a 3,186 $\mathrm{m}$ taxiing lane with the relevant buildings and infrastructures (see Fig. 1). DIA has consolidated its position as the largest international airport in Northeast China. In 2013, the number of passengers was approximately 16.05 million, with an average annual growth rate of $14.38 \%$. According to the development plans for DIA, passenger traffic will increase to 22 million by 2020 . However, the passenger capacity of DIA is only approximately 18 million, which means the airport would be in a significantly saturated state. Therefore, the Dalian government is facing the choice of either building a new runaway to enlarge DIA as soon as possible or building a new airport away from the main city zone to replace DIA. Considering the city's ecological environment, the final decision will require serious research.

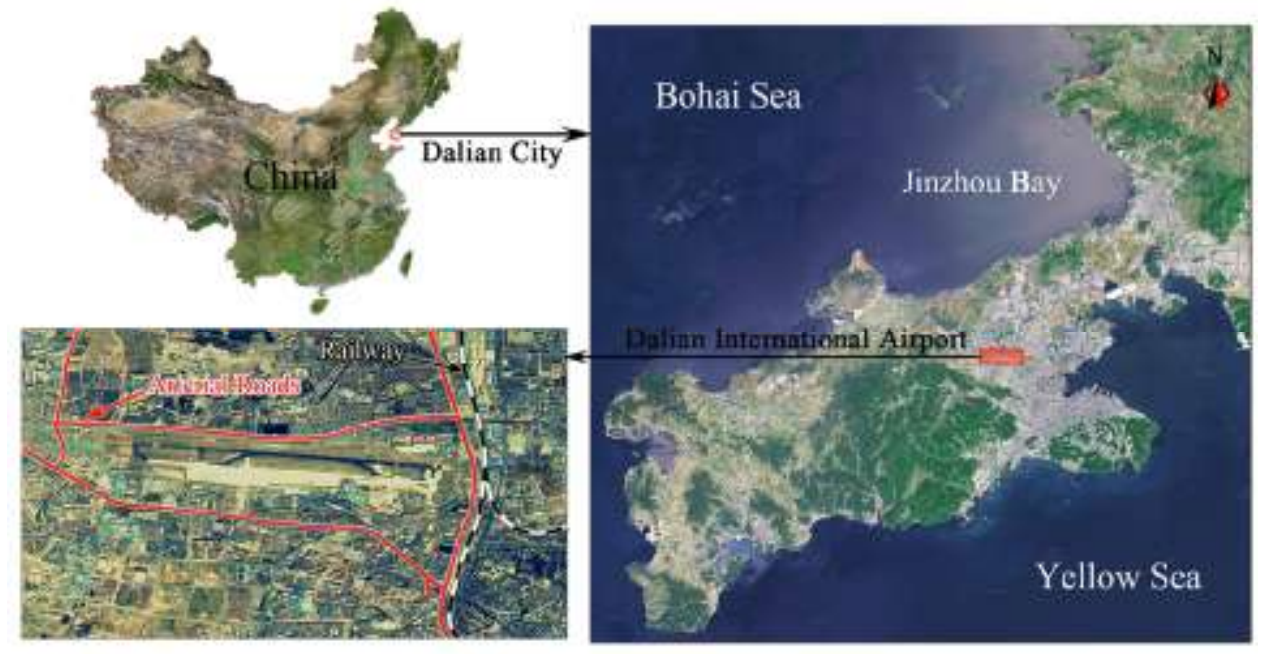

Fig. 1 Location of Dalian Zhoushuizi International Airport 


\section{Satellite data}

Landsat-8 data were used to retrieve the UHI. Landsat 8 (L8) was launched on February 11, 2013 and normal operations started on May 30, 2013. L8 has a ground track repeat cycle of 16 days with an equatorial crossing time at 10:00 a.m. The Operational Land Imager (OLI) on L8 (Table 1) is a nine-band push broom scanner with a swath width of $185 \mathrm{~km}$ with eight channels at $30 \mathrm{~m}$ and one pan-chromatic channel at 15-m spatial resolution. Compared to the Thematic Mapper (L4-5/TM) and the Enhanced Thematic Mapper Plus (L-7/ETM+) carried on previous Landsat missions, L8/OLI offers higher signal-to-noise ratios (SNRs) because of the longer integration times on the push broom scanner and a better quantisation (12 instead of 8 bits for radiometric digitisation) (Table 1). Orthorectified and terrain corrected Level 1T OLI imagery was obtained from USGS Earth Explorer (http://earthexplorer.usgs.gov/). Thermal Infrared Sensor (TIRS) has two bands, whose parameters are listed in Table 2.

Table 1

Comparisons of parameters between the L8/OLI bands and L7/ETM+ bands.

\begin{tabular}{|c|c|c|c|c|c|}
\hline \multicolumn{3}{|c|}{ L8/OLI } & \multicolumn{3}{|c|}{ L7/ETM+ } \\
\hline Band & $\begin{array}{l}\text { Wavelength } \\
\qquad(\mu \mathrm{m})\end{array}$ & $\begin{array}{c}\text { Spatial } \\
\text { Resolution } \\
\text { (m) }\end{array}$ & Band & $\begin{array}{l}\text { Wavelength } \\
\qquad(\mu \mathrm{m})\end{array}$ & $\begin{array}{c}\text { Spatial } \\
\text { Resolution } \\
\text { (m) }\end{array}$ \\
\hline 1(Coasatal/Aerosol) & $0.433-0.453$ & 30 & & & \\
\hline 2(Blue) & $0.450-0.515$ & 30 & 1(Blue) & $0.450-0.515$ & 30 \\
\hline 3(Green) & $0.525-0.600$ & 30 & 2(Green) & $0.525-0.605$ & 30 \\
\hline 4(Red) & $0.630-0.680$ & 30 & 3 (Red) & $0.630-0.690$ & 30 \\
\hline $5(\mathrm{NIR})$ & $0.845-0.885$ & 30 & 4(NIR) & $0.775-0.900$ & 30 \\
\hline 6(SWIR 1) & $1.560-1.660$ & 30 & 5(SWIR 1) & $1.550-1.750$ & 30 \\
\hline 7(SWIR 1) & $2.100-2.300$ & 30 & 7(SWIR 1) & $2.090-2.350$ & 30 \\
\hline $8(\mathrm{PAN})$ & $0.500-0.680$ & 15 & $8(\mathrm{PAN})$ & $0.520-0.900$ & 15 \\
\hline 9 (Cirrus) & $1.360-1.390$ & 30 & & & \\
\hline 10(Thermal 1) & $10.6-11.2$ & 30 & 61(Thermal 1) & $10.40-12.50$ & 60 \\
\hline 11(Thermal 2) & $11.5-12.5$ & 30 & 62(Thermal 2) & $10.40-12.50$ & 60 \\
\hline
\end{tabular}

Table 2

Load parameters of TIRS.

\begin{tabular}{ccccc}
\hline TIRS & $\begin{array}{c}\text { Central wavelength } \\
(\mu \mathrm{m})\end{array}$ & Lower limit $(\mu \mathrm{m})$ & Upper limit $(\mu \mathrm{m})$ & $\begin{array}{c}\text { Spatial Resolution } \\
(\mathrm{m})\end{array}$ \\
\hline Band-10 & 10.9 & 10.6 & 11.2 & 30 \\
Band-11 & 12.0 & 11.5 & 12.5 & 30 \\
\hline
\end{tabular}

\section{Methodology}

The calculation of at-sensor spectral radiance is a fundamental step in converting image data from multiple sensors and platforms into a physically meaningful common radiometric scale. We converted the Digital Numbers (DNs) for both reflective and thermal bands to at-sensor radiance using the following equation (Chander and Groeneveld, 2009):

$$
L_{\lambda}=\text { Gain } * \text { Pixelvalue }+ \text { Offset }
$$

where $L_{\lambda}$ is the spectral radiance at the sensor's aperture in $\mathrm{W} /\left(\mathrm{m}^{2} \mathrm{sr} \mu \mathrm{m}\right)$,

For an image-based atmospheric correction, a uniform atmospheric condition is usually assumed (Song et al., 2001), thus the correction will not have an effect on the analysis in this paper. Therefore, for all the reflective bands, we used the top-of-atmosphere (TOA) reflectance, which corrects for the 
cosine effect of solar zenith angles and changes in the exoatmospheric solar irradiance due to variation in the earth-sun distance. These variations can be significant geographically and temporally (Chander and Groeneveld, 2009). We computed the TOA reflectance according to the following equation:

$$
\rho_{\lambda}=\frac{\pi L_{\lambda} d^{2}}{E S U N_{\lambda} \sin \theta}
$$

Where: $L_{\lambda}$ is Radiance in units of $\mathrm{W} /(\mathrm{m} 2 * \mathrm{sr} * \mu \mathrm{m}) ; d$ is the distance between the Earth and sun, in astronomical units; $E S U N_{\lambda}$ is the solar irradiance in units of $\mathrm{W} /(\mathrm{m} 2 * \mu \mathrm{m})$; and $\theta$ is the sun elevation in degrees.

The temperature values obtained above were referenced to a blackbody, which is quite different from the properties of real objects. Therefore, a correction for spectral emissivity (e) is important. Each of the land use types was assigned an emissivity value. It was suggested that the emissivity values should be more detailed considering the nature of the land cover types. In this study, the vegetated area, built-up area, bare land, and water bodies were given the values of 0.95, 0.923, 0.92, and 0.9925, respectively (Artis and Carnahan, 1982; Masuda et al., 1988; Nichol, 1994).

Brightness Temperature: This option is only available for Landsat TM and ETM+ thermal imagery. Brightness temperatures (in Kelvin) are computed as follows:

$$
\mathrm{T}=\frac{K_{2}}{\ln \left(\frac{K_{1}}{L_{\lambda}}+1\right)}
$$

Where $K_{1}$ and $K_{2}$ are calibration constants, in Kelvin. ENVI reads these values from the Landsat metadata.

\section{Contribution of the airport to the UHI}

\section{Analysis method}

To study the contribution of the airport to the city UHI, two cloud-free Landsat-8 OLI images (Row/Path: 033/120 and 036/110) were used, which were collected on 11 August 2013 and 17 May 2013, respectively (Table 3). One image covers Dalian City, China, and the other covers Osaka, Japan. With the single-window algorithm, we retrieved the LST of Dalian's and Osaka's main areas. The retrieved results are shown in Fig. 2 and Fig. 3.

The thermal field was divided into five levels (Table 4) so that the high and low temperature areas can be identified.

Table 3

L8/OLI images of Dalian and Osaka.

\begin{tabular}{ccccc}
\hline \multicolumn{1}{c}{ Image } & Date & Time & Processor & City \\
\hline LC81200332013223LGN00 & 2013-08-11 & 02:30 GMT & LGPS 2.2.3 & Dalian \\
\hline LC81100362013137LGN01 & $2013-05-17$ & 02:50 GMT & LGPS 2.2.2 & Osaka \\
\hline
\end{tabular}

Table 4

Temperature levels of the LST.

\begin{tabular}{cc}
\hline Temperature level & The temperature range \\
\hline High temperature zone & $T_{s}>\mu+s d$ \\
High-medium temperature zone & $\mu+0.5 s d<T_{s} \leq \mu+s d$ \\
Medium temperature zone & $\mu-0.5 s d<T_{s} \leq \mu+0.5 s d$ \\
Medium-low temperature zone & $\mu-s d<T_{s} \leq \mu-0.5 s d$ \\
Low temperature zone & $T_{s} \leq \mu-s d$ \\
\hline
\end{tabular}




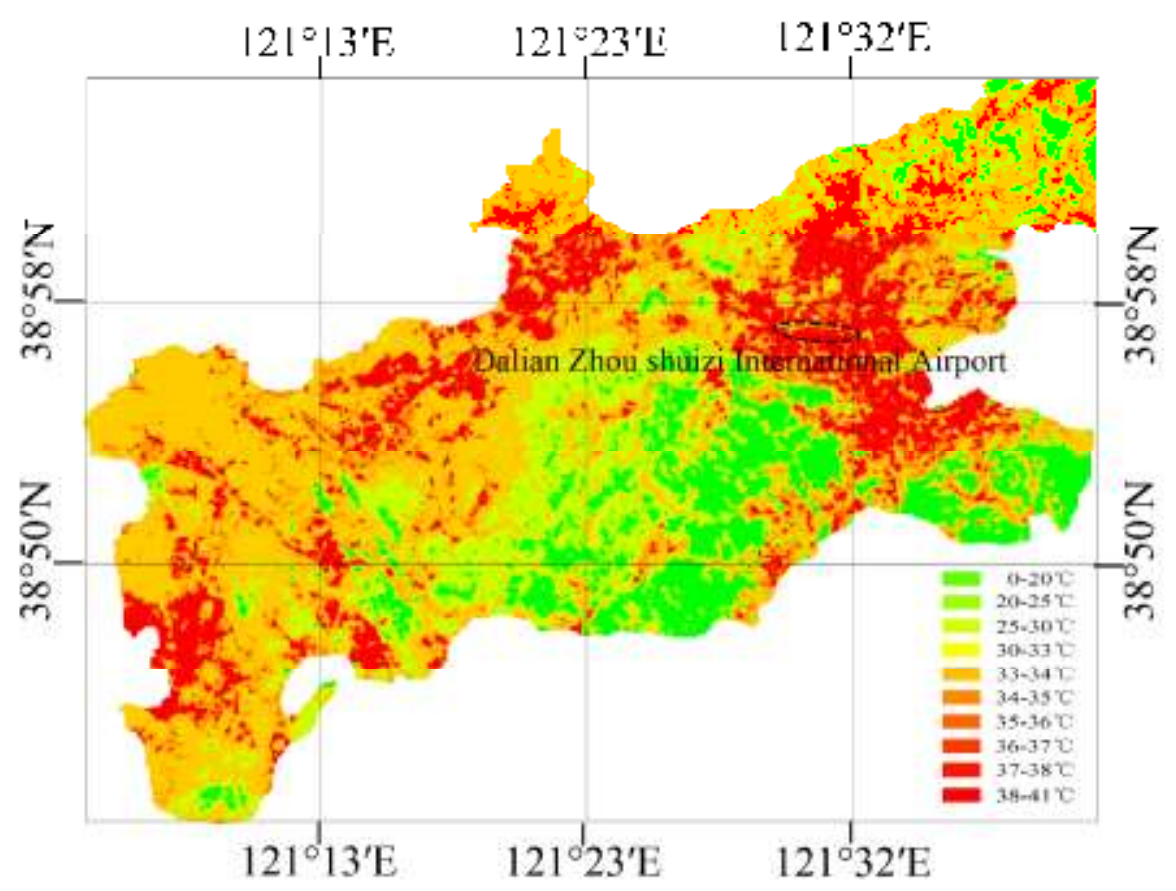

Fig. 2 Retrieved LST of Dalian's urban area

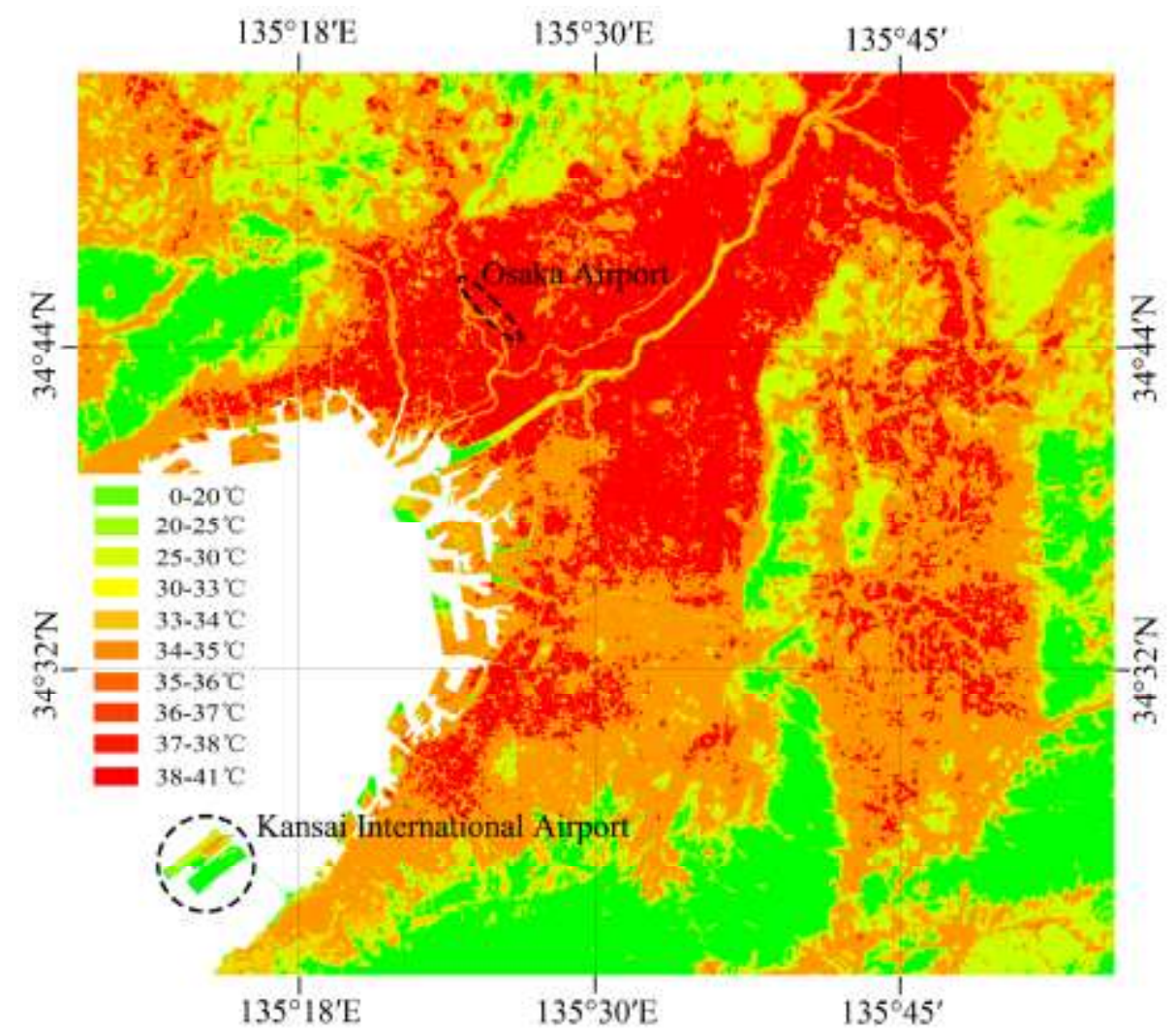

Fig. 3 Retrieved LST of Osaka's main area and Kansai Airport (offshore)

\section{Compared results}

The LST parameters of Dalian and Osaka are listed in Table 5. The thermal field range of Dalian's urban area and Osaka's area are listed in Table 6. 
Table 5

LST parameters of Dalian and Osaka $\left.\left(\mathrm{t}^{\circ} \mathrm{C}\right) / \mathrm{T}(\mathrm{K})\right)$.

\begin{tabular}{ccc}
\hline Statistics & Dalian & Osaka \\
\hline Minimum & $17.73 / 290.88$ & $4.12 / 277.27$ \\
Maximum & $41.87 / 315.02$ & $53.21 / 326.36$ \\
Mean & $28.88 / 302.03$ & $32.80 / 305.95$ \\
Standard deviation & $2.68 / 275.83$ & $5.74 / 278.89$ \\
\hline
\end{tabular}

Table 6

Temperature range of Dalian and Osaka $\left(t\left({ }^{\circ} \mathrm{C}\right) / \mathrm{T}(\mathrm{K})\right)$.

\begin{tabular}{ccc}
\hline Temperature levels & $\begin{array}{c}\text { Temperature range } \\
\text { of Dalian }\end{array}$ & $\begin{array}{c}\text { Temperature range } \\
\text { of Osaka }\end{array}$ \\
\hline High temperature zone & Ts $>31.56(304.71)$ & Ts $>38.54(311.69)$ \\
High-medium temperature zone & $31.22(304.37)<\mathrm{Ts} \leq 31.56(304.71)$ & $35.67(308.82)<\mathrm{Ts} \leq 38.54(311.69)$ \\
Medium temperature zone & $27.54(300.69)<\mathrm{Ts} \leq 30.22(303.37)$ & $(303.08) 29.93<\mathrm{Ts} \leq 35.67(308.82)$ \\
Medium-low temperature zone & $26.2(299.53)<\mathrm{Ts} \leq 27.54(300.69)$ & $(300.21) 27.06<\mathrm{Ts} \leq 29.93(303.08)$ \\
Low temperature zone & $\mathrm{Ts} \leq 26.2(299.35)$ & $\mathrm{Ts} \leq 27.06(300.21)$ \\
\hline
\end{tabular}

Dalian Zhoushuizi International Airport and Osaka Airport are in the high temperature zone, while Kansai International Airport is in the medium temperature zone (Table 7). Geographically, Dalian Zhou Shuizi International Airport and Osaka Airport are located in the central area of the city, while Kansai International Airport is located on the artificial island far away from the city, indicating that an offshore artificial island airport will significantly reduce the urban heat island compared to a land airport.

Table 7

LST parameters of different airports.

\begin{tabular}{cccc}
\hline Statistics & DIA & Osaka's Airport & Kansai International Airport \\
\hline Minimum & 27.98 & 36.13 & 17.63 \\
Maximum & 36.49 & 46.54 & 33.28 \\
Mean & 32.69 & 41.9 & 26.32 \\
Standard deviation & 1.98 & 1.82 & 3.55 \\
\hline
\end{tabular}

\section{Conclusions}

We retrieved the land surface temperatures (LSTs) of Dalian's and Osaka's main urban area using the thermal infrared band from Landsat- 8 data. The results show that Dalian Zhoushuizi Airport and Osaka Airport are in the high temperature zone and are the major contributors to the urban heat island effect.

Comparing the LST of Osaka's Kansai offshore airport and the Osaka airport, we found the average temperature of Kansai Airport is significantly lower than the average temperature of the Osaka airport, which shows an offshore airport can effectively reduce the urban heat island effect. 
According to the results above, the Dalian government has decided to build an offshore reclaimed airport in Jinzhou Bay. The offshore reclaimed airport is in an area of water of 5-8 m depth, has four runways and covers an area of $20 \mathrm{~km} 2$. The Dalian offshore airport would be the largest insular offshore airport in the world (see Fig. 4). The urban heat island effect produced by the Dalian offshore airport would be much less than the effect produced by Dalian Zhoushuizi Airport. The construction will improve the urban ecological environment.

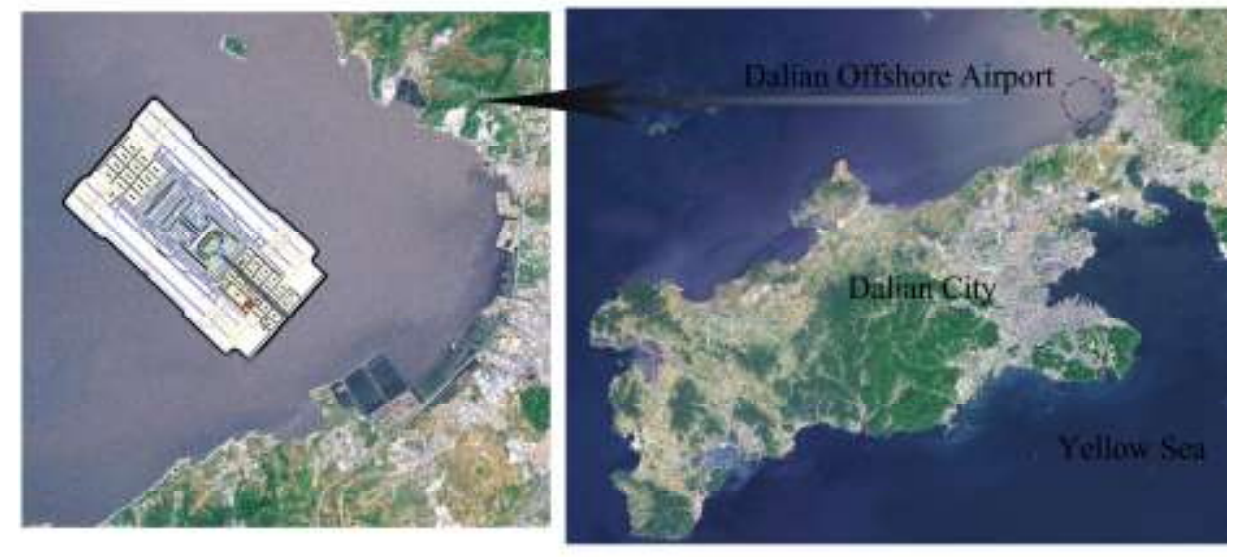

Fig. 4 The location of Dalian offshore artificial airport.

\section{References}

[1]Bentes, F.M., Heleno, T.A., \& Slama, J.G. 2013. Analysis of airport noise exposure around Viracopos International Airport using geographic information systems. Journal of Air Transport Management 31, 15-17.

[2]Carlson, T.N., \& Arthur, S.T. 2002. The impact of land use/land cover changes due to urbanization on surface microclimate and hydrology: A satellite perspective. Global and planetary changes 25, 49-56.

[3]Carlson, T.N., Perry, E.M., \& Schmugge, T.J. 1990. Remote estimation of soil moisture availability and fractional vegetation cover for agricultural fields. Agricultural and Forest Meteorology 52, 45-69.

[4]Chan, A.L.S. 2011. Developing a modified typical meteorological year weather file for Hong Kong taking into account the urban heat island effect. Building and Environment 46, 2434-2441.

[5]Chen, X.L., Zhao, H.M., Li, P.X., \& Yin, Z.Y. 2006. Remote sensing image-based analysis of the relationship between urban heat island and land use/cover changes. Remote Sensing of Environment 104, 133-146.

[6]Cheng, M.D., \& Corporan, E. 2010. A study of extractive and remote-sensing sampling and measurement of emissions from military aircraft engines. Atmospheric Environment 44, 4867-4878.

[7]Dai, X.Y., Zhang, L.Q., Guo, Z.Y., Wu, J.P., Li, X.D., \& Zhu, Y.L. 2009. Mechanism of formation of urban heat island effect and its spatial pattern in Shanghai. Acta Ecologica Sinica 29, 3995-4004.

[8]Feng, H.H., Zhao, X.F., Chen, F., \& Wu, L.C. 2013. Using land use change trajectories to quantify the effects of urbanization on urban heat island. Advances in Space Research 53, 463-473.

[9]Gallo K.P., Mcnab A.,Karl T.R., Brown J.F., Hood J.J., \& Tarpley J.D. (1993) The use of NOAA AVHRR data for assessment of the urban heat island effect. Journal of Applied Meteorology, 32(5): 899-908.

[10]Giannaros T.M., \& Melas D. (2012) Study of the urban heat island in a coastal Mediterranean City: The case study of Thessaloniki, Greece. Atmospheric Research, 118: 103-120. 
[11]Hu, L.Q., Brunsell, N.A. 2013. The impact of temporal aggregation of land surface temperature data for surface urban heat island (SUHI) monitoring. Remote Sensing of Environment 13, 4162-4174.

[12]Jusuf, S. K., Wong, N.H., Hagen, E., Anggoro, R., \& Hong, Y. 2007. The influence of land use on the urban heat island in Singapore. Habitat International 31, 232-242.

[13]Krüger, E., \& Emmanuel, R. 2013. Accounting for atmospheric stability conditions in urban heat island studies: The case of Glasgow, UK. Landscape and Urban Planning, 117:112-121.

[14]Kurniawan, J. S., \& Khardi, S. 2011. Comparison of methodologies estimating emissions of aircraft pollutants, environmental impact assessment around airports. Environmental Impact Assessment Review 31, 240-252.

[15]Lazzarini, M., Marpu, P.R., \& Ghedira, H. 2013. Temperature-land cover interactions: The inversion of urban heat island phenomenon in desert city areas. Remote Sensing of Environment $130,136-152$.

[16]Lee, T.W., Lee, J.Y., \& Wang, Z.H. 2012. Scaling of the urban heat island intensity using time-dependent energy balance. Urban Climate 2, 16-24.

[17]Li, J.J., Wang, X.R., Wang, X.J., Ma, W.C., \& Zhang, H. 2009. Remote sensing evaluation of urban heat island and its spatial pattern of the Shanghai metropolitan area, China. Ecological Complexity 6, 413-420.

[18]Li, J.X., Song, C.H., Cao, L., Zhu, F.G., Meng, X.L., \& Wu J.G. 2011. Impacts of landscape structure on surface urban heat islands: A case study of Shanghai, China. Remote Sensing of Environment 115, 3249-3263.

[19]Mackey, C. W., Lee, X.H., \& Smith, R. B. 2012. Remotely sensing the cooling effects of city scale efforts to reduce urban heat island. Building and Environment 49, 348-358.

[20]Mazaheri, M., Johnson, G.R., \& Morawska, L. 2011. An inventory of particle and gaseous emissions from large aircraft thrust engine operations at an airport. Atmospheric Environment 45, 3500-3507.

[21]McMillin, L.M. 1975. Estimation of sea surface temperature from two infrared window measurements with different absorption. Journal of Geophysical Research 20, 5113-5117.

[22]Pandey, P., Kumar, D., Prakash, A., Masih, J., Singh, M., Kumar, S., Jain, V.K., \& Kumar, K. 2012. A study of urban heat island and its association with particulate matter during winter months over Delhi. Science of the Total Environment 414, 494-507.

[23]Peace, H., Maughan, J., Owen, B., \& Raper, D. 2006. Identifying the contribution of different airport related sources to local urban air quality. Environmental Modelling \& Software 215, 532-538.

[24]Price, J. C. 1984. Land surface temperature measurements from the split window channels of the NOAA/AVHRR. Journal of geographical research 89, 7231-7237.

[25]Price, J.C. 1979. Assessment of the Urban Heat Island Effect through the Use of Satellite Data. Monthly Weather Review 107, 1554 -1557.

[26]Price, J.C. 1984. Land surface temperature measurements from split window channels of the NOAA7 advance very high resolution radiometer. Journal of Geophysical Research 89 7231-7237.

[27]Schafer, K., Jahn, C., Sturm, P., Lechner, B., \& Bacher, M. 2003. Aircraft emission measurements by remote sensing methodologies at airports. Atmospheric Environment 37, 5261-5271.

[28]Schwarz, N., Schlink, U., Franck, U., \& Großmann, K. 2012. Relationship of land surface and air temperatures and its implications for quantifying urban heat island indicators-An application for the city of Leipzig (Germany). Ecological Indicators 18, 693-704.

[29]Simões, A.F., \& Schaeffer, R. 2005. The Brazilian air transportation sector in the context of global climate change: $\mathrm{CO} 2$ emissions and mitigation alternatives. Energy Conversion and Management 46, 501-513. 
[30]Stettler, M.E.J., Eastham, S., \& Barrett, S.R.H. 2011. Air quality and public health impacts of UK airports. Part I: Emissions. Atmospheric Environment 45, 5415-5424.

[31]Streuker, D.K. 2002. A remote sensing study of the urban heat island of Huston, Texas. International Journal of Remote Sensing 23, 2595-2608.

[32]Streutker, D. R. 2003. Satellite-measured growth of the Urban Heat Island of Houston, Texas. Remote Sensing of Environment 85, 282-289.

[33]Takahashi, K., Yoshida, H., Tanaka, Y., Aotake, N., \& Wang, F. 2004. Measurement of thermal environment in Kyoto City and its prediction by CFD simulation. Energy and Buildings 16, 771-779.

[34]Unal, A., Hu, Y.T., Chang, M. E., Odman, M.T., \& Russell, A.G. 2005. Airport related emissions and impacts on air quality: Application to the Atlanta International Airport. Atmospheric Environment 39, 5787-5798.

[35]Upham, P., Thomas, C., Gillingwater, D., \& Raper, D. 2003. Environmental capacity and airport operations: current issues and future prospects. Journal of Air Transport Management, 9, 145-151.

[36]Vardoulakis, E., Karamanis, D., Fotiadi, A., \& Mihalakakou, G. 2013. The urban heat island effect in a small Mediterranean city of high summer temperatures and cooling energy demands. Solar Energy 94, 128-144.

[37]Velazquez-Lozada, A., Gonzalez, J.E., \& Winter, A. 2006. Urban heat island effect analysis for San Juan, Puerto Rico. Atmospheric Environment 40, 1731-1741.

[38]Voogt, J. A. \& Oke T. R. 2003. Thermal remote sensing of urban climates. Remote Sensing of Environment 86, 370-384.

[39]Weng, Q.H., Lu, D.S., \& Schubring, J. 2004. Estimation of land surface temperature-vegetation abundance relationship for urban heat island studies. Remote Sensing of Environment, 89, 467-483.

[40]Wilson, J. S., Clay, M., Martin, E., Stuckey, D., \& Vedder-Risch, K. 2003. valuating environmental influences of zoning in urban ecosystems with remote sensing. Remote Sensing of Environment 86, 303-321.

[41]Xiao, R.B., Ouyang, Z.Y., Zheng, H., Li, W.F., Schienke, E.W., \& Wang, X.K. 2007. Spatial pattern of impervious surfaces and their impacts on the surface temperature in Beijing, China. Journal of Environment Sciences 19, 250-256.

[42]Yan, H.K., Wang, N., Wei, L., \& Fu, Q. 2013. Comparing aircraft noise pollution and cost-risk effects of inland and offshore airports: The case of Dalian International Airport, Dalian, China. Transportation Research Part D 24, 37-43.

[43]Yuan, F., \& Bauer, M.E. 2007. Comparison of impervious surface area and normalized difference vegetation index as indicators of surface urban heat island effects in Landsat imagery. Remote Sensing of Environment 106, 375-386.

[44]Zhang H., Qi Z.F., Ye X.Y., Cai Y.B., Ma W.C., \& Chen M.N. (2013) Analysis of land use/land cover change, population shift, and their effects on spatiotemporal patterns of urban heat islands in metropolitan Shanghai, China. Applied Geography 44,121-133.

[45]Yin, Q., Zhu, S.Y., Gong, C.L. 2009. Remote sensing analysis of the relationships between daytime ground bright temperature and land-use types of city with shanghai as an example. Journal of Infrared and Millimeter Waves 02, 133-136.

[46]Sobrino, J.A., Jim nez-Mul̀oz, J.C., Paolini, L. 2004. Land surface temperature retrieval from Landsat TM 5. Remote Sensing of Environment 90, 434-440. 